\title{
A Standardized Implementation of Multicenter Quality Improvement Program of Very Low Birth Weight Newborns Could Significantly Reduce Admission Hypothermia and Improve Outcomes Within a Certain Region in China
}

Shu-yu Bi

Shandong Provincial Hospital, Shandong University

Yong-hui Yu ( $\square$ alice20402@126.com )

Shandong Provincial Hospital Affiliated to Shandong First Medical University

Cong Li

Liaocheng People's Hospital

Ping $X u$

Liaocheng People's Hospital

Hai-yan Xu

The First Affiliated Hospital of Shandong First Medical University

Jia-hui Li

The First Affiliated Hospital of Shandong First Medical University

Qiong-yu Liu

Women and Children's Healthcare Hospital of Linyi

Min Li

Women and Children's Healthcare Hospital of Linyi

Xin-jian Liu

Hebei PetroChina Central Hospital

Hui Wang

Hebei PetroChina Central Hospital

\section{Research Article}

Keywords: Very low birth weight, Neonates, Hypothermia, Quality improvement, Outcomes

Posted Date: November 10th, 2021

DOI: https://doi.org/10.21203/rs.3.rs-962766/v1 
License: (c) (i) This work is licensed under a Creative Commons Attribution 4.0 International License. Read Full License 


\section{Abstract}

Background: Admission hypothermia $\left(\mathrm{AH},<36.5^{\circ} \mathrm{C}\right)$ remains a major challenge for global neonate survival, especially in China. Due to high incidence of reginal $\mathrm{AH}$, we developed a prospective multicenter quality improvement $(\mathrm{QI})$ initiative to reduce regional $\mathrm{AH}$ and evaluate the impact on outcome among VLBW neonates.

Methods: The study used sequential Plan - Do - Study - Act (PDSA) approach. Clinical data were collected prospectively with 5 NICUs from Sino-Northern Neonatal Network (SNN) in China. Bundle come into practice since January 1, 2019. The clinical data in pre-QI phase (January 1, 2018- December 31, 2018) were compared with post-QI phase (January 1, 2019-December 31, 2020). Clinical characteristics and outcomes data were analysed.

Results: A total of 750 in-born VLBW infants were enrolled in the study, 270 in pre-QI period and 480 in post- QI period, respectively. There had no significant differences in clinical characteristics in two phases. Compared with pre-QI period, the percentage of AH decreased in the QI period $(95.9 \%$ vs $71.3 \%, P<0.01)$. Admission mod-severe hypothermia (AMSH) was improved significantly, reduced by $38.5 \%$ after QI (68.5 $\%$ vs $30 \%, \mathrm{P}<0.01)$. Average admission temperature improved after QI $\left[36.0^{\circ} \mathrm{C} \varangle 35.8^{\circ} \mathrm{C} \varangle 36.5^{\circ} \mathrm{C} \rrbracket v s 35.5^{\circ} \mathrm{C}\right.$ $\varangle 35.2^{\circ} \mathrm{C} \otimes 36.0{ }^{\circ} \mathrm{C} \nabla, \mathrm{P}<0.01$ ]. No significant increase in $\mathrm{AH}$ rate and thermal burns $(0.4 \% \mathrm{VS} 0 \%)$. Risks of mortality and late-onset neonatal sepsis (LOS) were significantly lower in post-QI period as compared to pre-QI period (aRR 0.19, 95\% $\mathrm{Cl} 0.09-0.39$; aRR 0.55, 95\% $\mathrm{Cl} 0.41-0.80$ ) whether adjusting for birth weight (BW), gestational age (GA) \small for gestational age (SGA), Apgar score at 5 min $<7$.

Conclusion囚lmplementation of multicenter thermoregulatory QI help in significant reduction of $\mathrm{AH}$ and AMSH of VLBW neonates within a certain area, which in turn can help to improve reginal neonatal outcomes. We gained a lot from QI, learned and explored a suitable method to continuous QI, this may provide reference for similar developing countries.

\section{Background}

With large surface area to body weight ratio, thin subcutaneous fat, poorly metabolic mechanisms, and inconspicuous heat consumption, very low birth weight $(<1500 \mathrm{~g}$, VLBW) infants are at high risk of heat loss [1-3]. Admission hypothermia $\left(\mathrm{AH},<36.5^{\circ} \mathrm{C}\right)$ is still common in infants born at hospitals $(32-85 \%)$ and homes (11-92\%), even in tropical environments, which remains a major challenge for global neonate survival, especially in developing countries like China [4-6]. It is shocked for us that we discovered the high proportion of AH of VLBW neonates in China during the process of collecting data, which was 89.3\% in 2017 and $88.2 \%$ in 2018 [5-6]. The incidence of AMSH is even more than 50\% [6]. Inadequate insulation measures, including usage of plastic membrane, preheated wool cap, temperature measurement and records, transportation incubator, monthly report of hypothermia situation, strategy of Plan-Do-Study-Act (PDSA) circles management strategy conducted less than $50 \%$ in northern China, 
besides, lack of attention paid by medical staff, and insufficient execution were also founded to be the main contributors [5-6].

Researches showed AH, especially AMSH is an independent risk factor for neonatal mortality and morbidity, connected with respiratory distress syndrome (RDS), intraventricular haemorrhage (IVH) and late-onset neonatal sepsis (LOS). [5, 7-11]. In 2014, a practical scientific point of view put forward, a theory of building in mechanisms to prompt reflection on your work, every day, seeking and incorporating feedback from others on team, began to be accepted by the public [12]. In order to improve outcomes, recognised quality improvement programs of temperature management for preventing hypothermia were rising internationally, and adjusted according to the actual situation of perinatal medical centers. With remarkable results and improved prognosis, the percentage internationally of $\mathrm{AH}$ in researches ranged in a low level from 10-30\% after QI [13-17]. A report from Indian made significant increasement in mean admission temperature of VLBW neonates $\left(35.3 \pm 0.6^{\circ} \mathrm{C}, 36.0 \pm 0.8^{\circ} \mathrm{C}\right.$, and $36.4 \pm 0.4^{\circ} \mathrm{C}$ during preintervention, intervention, and post-intervention phase) and improved composite neonatal adverse outcomes $(31 \%, 20 \%$, and $13.2 \%)$ by hypothermia QI project [15].

Even so, similar practice of internationally recognized hypothermia QI program has not been reported in China. How to apply and practice these advanced hypothermia quality improvement theories and methods in detail and ensure its efficiency in China is worthy to study. Therefore, an evidence-based regional hypothermia quality improvement is urgent to be developed to optimize thermoregulation and improve chinese regional perinatal healthcare quality in stabilization of VLBW neonates.

To optimize and standardize thermoregulation management process, we undertook a collaborative initiative for quality improvement project in 5 provincial and regional perinatal medical centers to explore the suitable way to improvement. One of our aims is to reduce incidence of $\mathrm{AH}$ by at least $10 \%$ over two years. Another is to evaluate the impact of this QI to outcomes on VLBW neonates within a certain area in China [18].

\section{Method}

\section{Study design and setting}

This prospective, multicentre cohort study was carried out over a period of 36months, from January 1 , 2018, to December 31, 2020, in 5 perinatal medical centers in northern China, which are all level 3 neonatal intensive care units (NICUs) with high population density to better homogeneity. The 5 recruited hospitals volunteered to participate in QI initiative, including 4 general hospitals and 1 maternal and child health care hospital, with averages of 34 and 30 beds in the neonatology departments and NICUs, respectively. The NICUs of the hospitals received an average of about 3536 newborns per year, of which VLBW infants were about 123 cases (3.5\%). The average ratio of nurses to bed and physician to nurse was about 1:1 and 1:2, respectively. 
Study was divided in two phases including pre-QI period (January 2018 to December 2018), and post-QI period (January 2019 to December 2020). In process of prospective data collection, the high incidence of $\mathrm{AH}$ was discovered in surprise for regular statistical analysis and month report of chart and graphics. We began to spend a lot of time on searching and reading literature, clinical studies and randomized controlled trials related to hypothermia to study and learn the physiological and pathological mechanism, adverse effects, prevalence and risk factors of hypothermia [1-4, 8-11,13-17,19-26]. By referring to scientific research methods from previous studies, we retrospectively investigated the temperature distribution and thermal insulation measures of 24 NICUs from northern China in 2017 to find out and distribution of $\mathrm{AH}$ and look into key drivers contributing to $\mathrm{AH}$ in processes including prenatal preparation, resuscitation, transport and post-entry into the NICU [18].

We assembled an interdisciplinary collaborative group named Hypothermia Clinical Research Group (HCRG) to develop initial bundle interventions by discussion and reading literatures and guidelines. The bundles were developed based on the medical literature review [20], the best practice recommendations of the California Perinatal Quality Care Collaborative (CPQCC) [21], the World Health Organization and the evidence-based principle of neonatal resuscitation projects [7,22-23].

\section{Interventions}

Interventions were confirmed in December 31, 2018, and came into use in January 1, 2019. A multidisciplinary team composed of medical and nursing staff from neonatology, obstetrics, and anesthesiology were established to implement QI practices. During QI period, we used Plan-Do-Study-Act (PDSA) methodology to adjust or expand interventions carried out to decrease $\mathrm{AH}$ [18]. We used the same digital laser infrared thermometer (OMRON, MC-347) for measurement in NICUs and made correction once a month to avoid errors. All the problems, suggestions, and temperature measurement videos were sent out to all participating centers by e-mail or WeChat discussion. Bundle emphasized accurate documentation of temperature at each point in time. All overall specific interventions were listed in Table 1 (Placed at the end of the article). Monthly random onsite visits for executive leader were built to face-toface communication for understanding barriers, requirement and supervising data quality. Regular inservice education and online lectures of videos of heat preservation were sent to public mailbox, which is acquired easily to help pediatric and obstetric providers and nurses strengthen awareness effectively and document temperature correctly.

\section{The outline of sequential PDSA cycles to adjust or expand interventions during QI phase}

\section{Initial Bundles (January 1-March 31, 2019):}

(1) Prenatal preparation (prenatal consultation, form multidisciplinary team, check materials);

(2)Set ambient temperature: Turn on the heating mode of the air conditioner and set the temperature above $25^{\circ} \mathrm{C}$; set radiant warmers at $34^{\circ} \mathrm{C}$

(3) Infant quickly dried after born; 
(4) Pre-warmed hats made of stockinette or wool was placed on the head;

(5) Weighed after being placed in a pre-warmed blanket;

(6) Using chemical preheated mattress;

(7) Document temperature at key time point (10 min after birth, arriving at the NICU, soon after every 30 min, till temperature $\geq 36.5^{\circ} \mathrm{C}$ ).

(8) Training and assessments on temperature measurement for nurses, making temperature measurement standard;

(9) Monthly charts reporting on hypothermia distribution and regularly quality control.

\section{PDSA Cycle 1 (April 1-May 31, 2019):}

Using polyethylene occlusive wrap infants without drying instead of drying infants immediately after birth.

\section{PDSA Cycle 2 (June 1-August 31, 2019):}

Introducing a heated transport incubator to keep warm in transportation.

\section{PDSA Cycle 3 (September 1,2019 - March 31, 2020):}

Revise admission hypothermia check list, adding individual signature blank area on check list to supervise effectively, and feedback checklist completeness at weekly meetings.

\section{PDSA Cycle 4 (April 1- December 31, 2020):}

Carrying out various online education lectures to medical staff and further emphasized the warmth link in the stabilization of Golden Hour for VLBW infants in NICUs by monthly online literature sharing learning.

The 4 cycles cover different links in the process of keeping warm, namely resuscitation, transportation, handover, and shared learning. In the process of resuscitation, especially for premature babies with small gestational age or small weight, the method of using plastic wrap to keep warm can better prevent the loss of water and heat through evaporation, radiation and convection. In addition to being economical, it can avoid the discomfort caused by the roughness of the repeatedly sterilized towel for wrapped child. Therefore, in order to better keep warm, we replaced the traditional method of wiping dry immediately after birth. Using polyethylene membrane with $30 \mathrm{~cm} \times 40 \mathrm{~cm}$ size to wrap babies, which could allow infants' head, torso and limbs be covered totally for better insulation [25-27].

In regular data feedbacks and interviews, we found great differences between different NICUs for transport warmth. Most units said they have not set special transport warmth part, but simply wrapped infants to the NICU. Transport distance varies with the actual situation of location between delivery room 
or operating room among units, some are on different floors of the same building, other are even in different buildings. To solve the problem, we introduced a heated transport incubator for transportation insulation in June 2019 [27-28], adding pre-transport preheating process in delivery room or operating room in bundle.

We found that the compliance of bundle declined in September 2019. The identified reasons of signal investigated by onsite visit were from insufficient staffing and inadequate force of supervision in handover. with relatively tense doctor-patient ratio in China, reducing turnover in staff is not feasible. To ensure the efficiency and feasibility of measures, the executive chairman of SNN suggested to revise the debriefing AH worklist for VLBW infants, adding signature blank to individual responsibility and feedback weekly according to local conditions in units. The changed version was tried on a small scale for 1 week for improved compliance, and was further promoted to all 5 perinatal medical centers. The revised paper version of worklist is listed as a member of necessary items. the list of prenatal necessary items is pasted on the side of the rescue box, as a warning to remind the pediatric consultation doctor to record the temperature in the delivery room in time. After being transferred to the NICU, the consultation staff fills in the general information and checks used thermal measures buttons in worklist, and then hand over it to the nurse on duty to complete the continuous measurement and recording work. Once worklist completed, it was clamped under the transparent plastic nurse work board. The next day the paper is retake by resident doctor, and feedback in every week meetings. The front and back patterns of the worklist paper were showed in Fig3-4.

During the fourth PDSA circle in March 2020, social factors such as social panic caused by epidemic situation, reduction of salary leading to the decrease of staff's work enthusiasm due to prevalence of Novel Coronavirus Epidemic. To rebuild awareness of hypothermia in medical staff, executive leader in SNN carried out various online education lectures to build up confidence and firm faith and further emphasized the warmth link in the stabilization of golden hour for VLBW infants by monthly online literature sharing learning and feedback meeting. In addition to emphasizing the compliance of measures, the meeting also emphasized the cooperation between obstetrics and pediatrics for prenatal communication, the cluster of management of medical operations, the standardized management of processes and the timeliness of feedback. We have added a link for prenatal consultation. Intrauterine consultation is carried out before delivery to carry out full communication between obstetrics and pediatrics. On the day of delivery, the consultation form is placed half an hour in advance and NICU is called at the same time to reserve sufficient time to reserve a transport incubator.

\section{Definitions}

Hypothermia was defined as a rectal temperature of less than $36.5^{\circ} \mathrm{C}$, according to the WHO [7]. Cold stress or mild hypothermia was defined as a temperature $36.0^{\circ} \mathrm{C}$ to $36.4^{\circ} \mathrm{C}$, moderate hypothermia was defined as a temperature $32.0^{\circ} \mathrm{C}$ to $35.9^{\circ} \mathrm{C}$, and severe hypothermia was defined as a temperature below $32{ }^{\circ} \mathrm{C}$. Normothermia was defined as a body temperature between $36.5^{\circ} \mathrm{C}$ to $37.5^{\circ} \mathrm{C}$. Redirection of intensive care was defined as limited care (not intensifying medical treatment) or withdrawal of care [29]. 
Composite outcome included death before discharge or any of major morbidity including grade 3 or 4 IVH, grade 3 or 4 ROP and stage 2 or higher NEC (Bell et al) according to the Practice of Neonatology (5th Edition) [30]. LOS was diagnosed by the clinical manifestations of systemic infection after 3 days of birth and abnormal values for 2 or more of the following non-specific infection indicators: WBC $<5 \times 109 / \mathrm{L}$ or WBC > $20 \times 109 / \mathrm{L}$; C-reactive protein (CRP) $\geq 10 \mathrm{mg} / \mathrm{L}$; platelets (PLTs) $\leq 100 \times 109 / \mathrm{L}$; and procalcitonin $(\mathrm{PCT})>2 \mathrm{ng} / \mathrm{ml}$ [31]. Moderate and severe BPD was defined as the requirement of any inspired fraction oxygen above 0.21 at the corrected GA of 36 weeks [32]. If any fresh blood appears in the trachea intubation, with hematocrit dropping by more than $10 \%$ in the blood routine examination and the decrease of transmittance on the chest X-ray was diagnosed Pneumorrhagia [33].

\section{Data extraction}

The admission temperatures related data of VLBW infants were collected prospectively in SNN. The database provided maternal, delivery, neonatal clinical materials and temperature data before discharge, and the data were collected by a standardized operating procedure $[5,18,34]$. The admission temperature was defined as the infant's rectal temperature measured at admission to the NICU within $1 \mathrm{~h}$ after birth, because it's closer to core temperature [35]. A worklist of temperature evaluation list for VLBW infants was used to carry on hand by the consulting physician before every intrapartum consultation to data collection, which documenting interventions on the back side and temperature data at different time points after birth on the front side in Fig.3-4.

\section{Statistical analysis}

Demographic data are expressed as medians and interquartile ranges (IQR). Categorical data are shown as percentages. Comparison of clinical characteristics between groups was calculated with the MannWhitney U-test and Chi-square test. Risks of outcomes were compared between groups were tested in a bivariate analysis, followed by a logistic regression analysis. $\mathrm{P}<0.05$ was considered statistically significant. The statistical analyses were conducted using SPSS v. 26.0 (SPSS Inc., Chicago, Illinois) and QI Macros 2018.09 (Denver, CO). Special cause signals were identified by using standard control chart rules [36].

\section{Results}

A total of 890 in-born infants with VLBW neonates were enrolled in the study; 49 infants were excluded because they were out-born; 20 infants were excluded because their mother had a fever during delivery (temperature $\geq 38.4^{\circ} \mathrm{C}$ ). Additionally, 64 infants with redirection of intensive care and 7 infants with missing temperature data were excluded. The remaining 750 VLBW infants were included in this analysis, 270 in pre-QI period and 480 in post-QI period, respectively. (Fig 1). Infants born during both periods had no significant differences in neonatal and perinatal clinical characteristics, including GA, gender, BW, polyembryony, caesarean section, the percentage of 5 min Apgar scores $₫ 7$ and so on(Table 2). Proportion of rectal temperature $\geq 36.5^{\circ} \mathrm{C}$ within first hour after admission during QI phase decreased month by month steadily (Fig 2). 


\section{Hypothermia}

Table 3 shows the distribution of $\mathrm{AH}$ in two periods. Compared with pre-QI period, the percentage of $\mathrm{AH}$ in VLBW infants decreased in the QI period $(95.9 \%$ vs $71.3 \%, \mathrm{P}<0.01)$. AMSH was improved significantly, reduced by more than a half after QI $(68.5 \% \mathrm{vs} 30 \%, \mathrm{P}<0.01)$. Infants in post-QI phase had higher average admission temperature $\left[36.0^{\circ} \mathrm{C} \varangle 35.8^{\circ} \mathrm{C} \rrbracket 36.5^{\circ} \mathrm{C} \rrbracket\right.$ vs $\left.35.5^{\circ} \mathrm{C} \varangle 35.2^{\circ} \mathrm{C} \varangle 36.0^{\circ} \mathrm{C} \rrbracket, \mathrm{P}<0.01\right]$. There was no significant increase in the rate of hyperthermia (0.4\%VS $0 \%)$. No thermal burns were reported. Proportion of $\mathrm{AH} \geq 36.5^{\circ} \mathrm{C}$ within first hour after admission during QI phase increased month by month (Fig.2). Risks of AH and AMSH decreased significantly in post-QI phase (aRR:0.10, 95\% Cl 0.050.19; aRR:0.19, 95\% $\mathrm{Cl} 0.13-0.26$ ) whether adjusting for BW, GA囚SGA, Apgar score at 5 min $<7$ (Table 5). The control $P$ chart revealed that central line was shifting down during post-QI phase, from a baseline of $95.9 \%$ to $71.6 \%$, which is consistent with temperature distribution above (Fig 5). The improvement is still ongoing in chart.

\section{Outcomes}

Compared with pre-QI phase, the mortality of VLBW neonates declined obviously in post-QI phase, from $10.7 \%$ to $2.7 \%(P<0.01)$ (Table 4$)$. the incidence of LOS also felled by $11 \%$ obviously in post-QI phase, from $33 \%$ to $22 \%(P<0.01)$ (Table 4). Compared to pre-QI period, the unadjusted relative risk (RR) of mortality and LOS for post-QI period was separately in reduction to $0.23,95 \% \mathrm{Cl} 0.12-0.45$ and $0.55,95 \%$ Cl 0.41-0.80 (Table 4). These remained significant after adjusting for confounding factors including BW, GA凶SGA, and Apgar score at 5 min $<7$ (aRR 0.19, 95\% Cl 0.09-0.39; aRR 0.56, 95\% Cl 0.40-0.80)(Table 5 ). We did not find any difference in composite outcome, incidence of NEC ( $\geq$ stage 2$)$, BPD ( $\geq$ stage 2 ), IVH ( $\geq$ grade 3 ), and ROP ( $\geq$ grade 3 ) during two phases whether adjustment. (Table 5 ).

\section{Discussion}

with such a high incidence of hypothermia in baseline, it is surprising that substantial improvement can be achieved on reduction of $\mathrm{AH}$ and neonatal outcomes with the initiation implementation of a multicener regional quality improvement program. The risk of overheating is not increasing. By standardizing thermoregulate management procedure, awareness of hypothermia among staff was emphasized to some extent, which accidentally made it an invisible intervention to contribute to on-going improvement.

with unbalanced medical resources and a large gap between urban and rural areas, hypothermia in developing countries is still a serious situation and a major challenge to newborn survival worldwide [4]. Researches showed $\mathrm{AH}$ is an independent risk factor for neonatal mortality and morbidity, associated with a high likelihood of IVH and LOS [5]. Recognizing the current state of hypothermia in units is conducive to sounding the alarm to medical personnel, prompting them to make efforts to find the cause, strengthen the awareness of disease, contributed to implementing targeted clinical quality improvement, accordingly, enhancing quality of care and improving clinical neonatal outcomes $[13-17,25,37]$. 
Countries are working to standardize temperature management procedures by QI to reduce hypothermia and improve outcomes [13-17,37]. A QI study from a single center in Indian for 6 months shows overall admission hypothermia decreased by QI from $82 \%$ to $45 \%$, and moderate hypothermia reduced from $46 \%$ to $<10 \%(P<0.001)$ with significant reduction in incidence of IVH (13\% Vs $4.7 \%)$, LOS (38\% Vs $19 \%)$ and metabolic acidosis (43\% Vs $28 \%$ ) [37]. Another QI research from Singapore reported that Incidence of AH decreased from 79.4 to $40.5 \%(P<0.001)$, constituting a 49\% improvement $(\mathrm{OR}=0.177,95 \% \mathrm{Cl}$ : $0.099-$ 0.316), though IVH and mortality remained unchanged [14]. In our study, the mortality and incidence of LOS of VLBW neonates declined obviously in post-QI phase, which is consistent with previous studies. However, we did not find any difference in composite outcome, especially IVH ( $\geq$ grade 3 ) in two groups. On the one hand, the results may differ due to different external environmental conditions, geographical location, climatic factors and race of the studies. Secondly, the sample size in study may not large, which may cause biased accuracy from sampling error. Nevertheless, the improvement is still ongoing, the effect may be apparent in continuous QI. On the other hand, because lack of synergistic effect of multicenter researches, it may not be comparable because previous studies are single-center studies.

The main advantage of this study is that objectively showing a successful practice for targeted multicenter AH quality improvement of VLBW infants in northern China. Prospective, and substantial sample multicenter research provides the credibility of result. It also contributes to the global epidemiology of hypothermia, indicating that hypothermia remains a major challenge in developing countries, especially in China. Initiation in action and awareness in thinking are key parts to promote a shift in the improvement process $[7,13-17,25,37]$. Most importantly, the study provides a method to figure out issues instantly and find solutions, which may promote continuous quality improvement. The supervision and training of employees increase the possibility of a standardized temperature measurement, it can improve accuracy to the greatest extent and to avoid bias. Random spot visits facilitated the supervision of each unit and contributed to timely identification of the obstacles encountered in the improvement process. There were no obvious changes in possible confounders, including patient factors or the resuscitation condition, as a result, decrease in hypothermia is probably attributable to the thermoregulation bundle in QI.

If our approach produces similar results elsewhere, it could add significant value to neonatal care. We have not quantified the material cost of the thermoregulatory bundle, because its economy and applicability are visible. Polyethylene wrapping are much cheaper than medical-grade bags [24-27]. All the interventions could be conducted easily and at a low cost, which is allowed to reference, copy, and apply. In general, improving quality (compliance of process) can increase the value of interventions without increasing costs. Neither a lower Apgar score nor a more intensive recovery was observed before and after QI, indicating that there was no significant change in opportunity cost.

Besides, how to maximize the effectiveness of each intervention depends on the good compliance and execution and feedback of thermal insulation measures [7, 25, 29,15-16, 24-27]. In our study, we pay more attention to the consistency and completeness of the implementation of measures. We monitored the compliance of general measures implemented every month and found that the average compliance of 
general measures in 2019 was $68.0 \%$. The trend is more stable in 2020 , with a large-scale improvement, which is $92.0 \%$. And it is interesting that the conversation between executive chairman and NICU department directors during onsite visits showed that the biggest obstacle is that they did not want to break out of their comfort zone (systematic clinical habits or conventions) to make changes, in their perspectives, it is difficult to strictly implement bundle in such a large population country with tight human resources at the beginning. In addition to the daily tedious work, medical staff is hard to pay extra unrewarded energy and physical labor to increase workload. Maybe this deep-rooted mentality is one of the huge difficulties to change, which is of primary importance for next plan to ensure the continuous improvement of the effect. For two-year-effort, it is lucky for us to make progress through regular data monitoring and regular reporting, monthly online meetings for online discussions. Nonetheless, it is happy to see the decline of $\mathrm{AH}$ and improvement of neonatal outcomes, because it means hypothermia began to be paid attention in northern China from a certain point of view.

A possible limitation of this study is its differences between NICUs. We standardized the temperature measurement methods and instruments, conducted continuous temperature detection and feedback regularly. However, variation in execution capability of thermal measures in NICUs likely contributed to different center outcomes. We didn't evaluate the changes. Another is that the total number of observation cases has decreased in 2020 (about two-thirds of the cases in 2019), which may affect our interpretation of the results, but we focus on comparing the general AH proportion between groups, so the result is still has certain representativeness.

Potential reasons of occasional residual severe hypothermia events may be related to multiple births in the operating room, prolonged asphyxiation and resuscitation, and insufficiency of preparation for emergency delivery, which further remind us to pay more attention to these areas in the next phase. Areas for future review include strengthening supervision in compliance of interventions, development of leadership training of academic leaders in units in order to maximize the autonomy of each NICUs.

In developing countries, the consciousness of keeping warm is very important. We expected our initial exploration of hypothermia quality improvement can give inspiration and lesson to units or readers who were suffered from similar condition. Although there were still shortcomings, we had gained a lot in the QI process to constant intercommunication, learning and sharing, furthermore, achieved our goal to reduce $\mathrm{AH}$ in the end.

\section{Conclusions}

By drawing on international experience and combined with China's regional characteristics, we have explored a befitting path of reducing AH by QI through two years of effect, proving its feasibility and effectiveness through clinical practice. We learned how to find problems from the data, identify obstacles, and make targeted changes, what's more, we successfully aroused the attention of perinatal medical staff to hypothermia in region, which may be of reference to a certain extent. 


\section{Abbreviations}

VLBW: Very low-birth weight; QI: Quality improvement; PDSA: Plan-do-study-act; NICU: Neonatal intensive care unit; $\mathrm{AH}$ : Admission hypothermia; AMSH: admission mod-severe hypothermia; HCRG: Hypothermia Clinical Research Group; CPQCC: California Perinatal Quality Care Collaborative; IQR: interquartile ranges; GA: Gestational age; BW: Birth weight; SGA: Small for gestational age; RR: relative risk; aRR: adjusted relative risk; IVH: Intraventricular haemorrhage; NEC: Necrotizing enterocolitis; LOS: Late-onset neonatal sepsis; BPD: Bronchopulmonary dysplasia; ROP: Retinopathy of prematurity;

\section{Declarations}

Ethics approval and consent to participate: The research protocol has been ethically reviewed by the Ethics Committee of the Provincial Hospital Affiliated to Shandong First Medical University (LCYJ: No. 2019-004). Informed consent to participate in the study have been obtained from participants' parent.

Consent for publication: Not Applicable.

Availability of data and materials: The data that support the findings of this study are available from the corresponding authors upon reasonable request.

Conflict of interest: No financial or nonfinancial benefits have been received or will be received from any party related directly or indirectly to the subject of this article.

Funding: The National Assisted Reproductive and Eugenics Engineering Technology Research Center and Key Laboratory of Reproductive Endocrinology Ministry of Education Open Project, 2017.11-2022.10 covered all the costs for data collection instruments, data collection, data entry and payments for supervisors and advisers. The funder of our study is the corresponding author of this study, professor Yong-hui Yu. She is responsible for designing research, interpreting results and revising manuscripts in this study.

Authors' contributions: Yong-hui Yu, the corresponding author, doctorate, and professor of medicine, designed the study, trained and supervised the data collectors, interpreted the results and revised the manuscript. The first author, namely, SYB, played a role in the analysis and interpretation of the data and in preparing and drafting the manuscript. The co-first authors, namely, CL, PX, HY-X, JH-L, QY-L, ML, XJ-L, and HW participated in the design of the study, the collection and interpretation of the data and writing the manuscript. All authors listed on the manuscript approved the submission of this version of the manuscript and take full responsibility for the manuscript.

Acknowledgements: We would like to thank Li Wang from the First Affiliated Hospital of Sun Yat-sen University, Guangzhou, China for professional supports and Xiao-chen Shu, Professor of Statistics, Soochow University for statistics and methodology assistance and guidance with this research project.

\section{Author information}


All co-first authors: Shu-yu $\mathrm{Bi}^{1}$, Yong-huiY $\mathrm{u}^{1,2^{*}}$, Cong $\mathrm{Li}^{3 \dagger}$, Ping $\mathrm{Xu}^{3}$, Hai-yan $\mathrm{Xu}^{4 \dagger}$, Jia-hui $\mathrm{Li}^{4}$, Qiong-yu $\mathrm{Liu}^{5+}$, Min Li ${ }^{5}$, Xin-jian Liu ${ }^{6 \dagger}$, and Hui Wang ${ }^{6}$

1.Department of Neonatology, Shandong Provincial Hospital, Cheeloo College of Medicine, Shandong University, Jinan 250021, Shandong, China. E-mail: bsy691564538@sina.com. 2.Department of Neonatology, Shandong Provincial Hospital Affiliated to Shandong First Medical University, Jinan 250021, China. E-mail: alice20402@126.com. 3. Department of Neonatology, Liaocheng People's Hospital, Liaocheng 252000, Shandong, China. E-mail: 2682653862@qq.com. 4.Department of Neonatology, The First Affiliated Hospital of Shandong First Medical University, Jinan 250014, Shandong, China. E-mail: 2426296683@qq.com. 5.Department of Neonatology, Women and Children's Healthcare Hospital of Linyi, Linyi 276000, Shandong, China. E-mail: liuqyu@163.com. 6.Department of Neonatology, Hebei PetroChina Central Hospital, Langfang 065000, Hebei, China. E-mail: drliu7285@163.com.

\section{References}

1. Aylott M. The neonatal energy triangle. Part2: Thermoregulatory and respiratory adaption. Paediatr Nurs. 2006; doi:10.7748/paed.18.7.38.s28.

2. Capobianco JA. Keeping the newborn warm: how to safeguard the infant against life-threatening heat loss. Nursing. 1980;10:5.

3. Thomas K. Thermoregulation in neonates. Neonatal Netw. 1994;13:22.

4. Lunze K, Bloom DE, Jamison DT, Hamer DH. The global burden of neonatal hypothermia: systematic review of a major challenge for newborn survival. BMC Med. 2013; doi:10.1186/1741-7015-11-24.

5. Yu YH, Wang L, Huang L, Wang LL, Huang XY, Fan XF, et al. Association between admission hypothermia and outcomes in very low birth weight infants in China: a multicentre prospective study. BMC Pediatr. 2020; doi: 10.1186/s12887-020-02221-7.

6. Yong-hui Yu, Shandong Multicenter Study Coordination for Admission Hypothermia in Neonatal Intensive Care Units. Hypothermia on admission in both very low and extremely low birth weight infants in Shandong Province: a multicenter survey. Chin J Perinat Med. 2019; doi: 10.3760/cma.j.issn.1007-9408.2019.08.004(in Chinese).

7. World Health Organization, Department of Reproductive Health Research. Thermal protection of the newborn: a practical guide.Geneva: Division of Reproductive Health (Technical Support), World Health Organization; 1997. p. 56.

8. Hammarlund K, Sedin G. Transepidermal water loss in newborn infants. Vl. Heat exchange with the environment in relation to gestational age. Acta Paediatr Scand. 1982; doi: 10.1111/j.16512227.1982.tb09398.x.

9. Costarino A, Baumgart S. Modern fluid and electrolyte management of the critically ill premature infant. Pediatr Clin North Am. 1986; doi: 10.1016/s0031-3955(16)34974-4.

10. Lyu Y, Shah PS, Ye XY, Warre R, Piedboeuf B, Deshpandey A, et al; Canadian Neonatal Network. Association between admission temperature and mortality and major morbidity in preterm infants 
born at fewer than 33 weeks' gestation. JAMA Pediatr. 2015; doi:

10.1001/jamapediatrics.2015.0277.

11. Laptook AR, Salhab W, Bhaskar B; Neonatal Research Network. Admission temperature of low birth weight infants: predictors and associated morbidities. Pediatrics. 2007; doi: 10.1542/peds.20060943.

12. Fogarty CT, Mauksch LB. "That's why they call it practice". Fam Syst Health. 2014; doi: $10.1037 / \mathrm{fsh} 0000093$.

13. Russo A, McCready M, Torres $L$, Theuriere $C$, Venturini S, Spaight $M$, et al. Reducing hypothermia in preterm infants following delivery. Pediatrics. 2014; doi: 10.1542/peds.2013-2544.

14. Yip WY, Quek BH, Fong MCW, Thilagamangai, Ong SSG, Lim BL, et al. A quality improvement project to reduce hypothermia in preterm infants on admission to the neonatal intensive care unit. Int $\mathrm{J}$ Qual Health Care. 2017; doi: 10.1093/intqhc/mzx131.

15. Sharma D, Murki S, Kulkarni D, Pawale D, Vardhelli V, Anne RP, et al. The impact of a quality improvement project to reduce admission hypothermia on mortality and morbidity in very low birth weight infants. Eur J Pediatr. 2020; doi: 10.1007/s00431-020-03711-7.

16. Harer MW, Vergales B, Cady T, Early A, Chisholm C, Swanson JR. Implementation of a multidisciplinary guideline improves preterm infant admission temperatures. J Perinatol. 2017; doi: 10.1038/jp.2017.112.

17. Russo A, McCready $M$, Torres $L$, Theuriere $C$, Venturini $S$, Spaight $M$, et al. Reducing hypothermia in preterm infants following delivery. Pediatrics. 2014; doi: 10.1542/peds.2013-2544.

18. Wang L, Yu Y-h, et al. Shandong Multicenter Study Coordination for Admission Hypothermia in Neonatal Intensive Care Units. Evidence-based practice for improving quality to to reduce the incidence of admission hypothermia: a multicentered study protocol. Chin J Based Pediatr. 2019; doi:10.3969/j.issn.1673-5501.2019.02.012(in Chinese).

19. Fawcett K. Preventing admission hypothermia in very low birth weight neonates. Neonatal Netw. 2014; doi: 10.1891/0730-0832.33.3.143.

20. Bell R, Finer N, Halamek L,et al.California Perinatal Quality Care Collaborative Delivery Room Management Quality Improvement Toolkit[EB/OL].(2011-06)[2018-12-01].www. cpqcc.org/sites/default/files/DRToolkitUpdateFinal5-18-11. pdf.

21. Wyckoff MH, Aziz K, Escobedo MB, et a1. Part 13: Neonatal Resuscitation:2015 American Heart Association Guidelines Update for Cardiopulmonary Resuscitation and Emergency Cardiovascular Care[J]. Circulation, 2015, 132(18 Suppl 2): S543-560. doi: 10.1161/CIR.0000000000000267.

22. Expert Group on China's Neonatal Resuscitation Project. Guidelines for China's Neonatal Resuscitation (Beijing Revised 2016) [J]. Chin J Perinat Med. 2016; doi: 10.3760/cma.j.issn.10079408.2016.07.001.

23. Qian Miao, Sha Li, Effects of quality improvement in delivery room resuscitation on very or extremely low birth weight infants, Chin J Perinat Med, 2017; doi: 10.3760/cma.j.issn.1007-9848.2017.05.010. 
24. Ping Lu, Li-Ping Zhang, A randomized controlled study of the effect of intelligent carbon fiber infant thermal insulation wrap on the in-hospital transport thermal insulation of very low birth weight infants, Chin J Perinat Med, 2014, 17:1(in Chinese).

25. Deepak Sharma. Golden Hour of Neonatal Life: Need of the Hour,Sharma Maternal Health, Neonatology, and Perinatology. 2017; 3:16.

26. Rohana J, Khairina W, Boo NY, Shareena I. Reducing hypothermia in preterm infants with polyethylene wrap. Pediatr Int. 2011; doi: 10.1111/j.1442-200X.2010.03295.x.

27. Singh A, Duckett J, Newton T, Watkinson M. Improving neonatal unit admission temperatures in preterm babies: exothermic mattresses, polythene bags or a traditional approach? J Perinatol. 2010; doi: $10.1038 /$ jp.2009.94.

28. McNellis EM, Leonard AR, Thornton KA, Voos KC. Improving Thermal Support in Very and Extremely Low Birth Weight Infants during Interfacility Transport. Pediatr Qual Saf. 2019; doi: 10.1097/pq9.0000000000000170.

29. Eventov-Friedman S, Kanevsky H, Bar-Oz B. Neonatal end-of-life care: a single-center NICU experience in Israel over a decade. Pediatrics. 2013; doi: 10.1542/peds.2012-0981.

30. chlapbach LJ, Graf R, Woerner A, Fontana M, Zimmermann-Baer U, Glauser D, et al. Pancreatic stone protein as a novel marker for neonatal sepsis. Intensive Care Med. 2013; doi: 10.1007/s00134-0122798-3.

31. Jobe AH, Bancalari E. Bronchopulmonary dysplasia. Am J Respir Crit Care Med. 2001; doi: 10.1164/ajrccm.163.7.2011060. PMID: 11401896.

32. Lin TW, Su BH, Lin HC, Hu PS, Peng CT, Tsai CH, Liang WM. Risk factors of pulmonary hemorrhage in very-low-birth-weight infants: a two-year retrospective study. Acta Paediatr Taiwan. 2000; 41:5.

33. Choi CW, Park MS. Data Management and Site-Visit Monitoring of the Multi-Center Registry in the Korean Neonatal Network. J Korean Med Sci. 2015; doi: 10.3346/jkms.2015.30.S1.S19.

34. Ogrinc G, Davies L, Goodman D, Batalden P, Davidoff F, Stevens D. SQUIRE 2.0 (Standards for Quality Improvement Reporting Excellence): revised publication guidelines from a detailed consensus process. BMJ Qual Saf 2015; doi:10.1136/bmjqs-2015-004411.

35. Patodia J, Mittal J, Sharma V, Verma M, Rathi M, Kumar N, Jain R, Goyal A. Reducing admission hypothermia in newborns at a tertiary care NICU of northern India: A quality improvement study. $\mathrm{J}$ Neonatal Perinatal Med. 2021; doi: 10.3233/NPM-190385.

\section{Tables}


Bundle of Temperature management procedure at NICUs

\section{Prenatal preparation}

1. Prenatal preparation (prenatal consultation, form multidisciplinary team, check materials)

2.Set the ambient temperature above $25^{\circ} \mathrm{C}$, set radiant warmers at $34^{\circ} \mathrm{C}$

3.Prewarm the hat

4.Prepare a polyethylene wrap

5. Push a heated transport incubator forward to delivery or operating room, plug it in and keep charging, switch on incubator and set it to target temperature range $: 36-36.5^{\circ} \mathrm{C}$

\section{Resuscitation euthermia}

6.Preheated blankets wrapping after birth

7.Quickly weight after being placed in a pre-warmed blanket

8.Infant immediately after birth wrapped with a polyethylene wrap without drying

9.Place a pre-warmed hat on the head

10.Resuscitation under chemical preheated mattress

11.Document temperature at 10 min after birth

\section{Transportation euthermia}

12. Put infants into a heated transport incubator and start transportation

\section{After admission to NICU}

13.Preheat daily materials in incubator (diapers, oxygen probe, stethoscope)

14.Put the infants into the incubator immediately when arriving at NICU

15. Document temperature continuously within one hour after birth

16. Retest temperature when arriving at the NICU, soon after every 30 min

17. Document the time point when temperature at $\geq 36.5^{\circ} \mathrm{C}$

18.Nuring and medical operations are centralized implemented

19.Training and assessments on temperature measurement for nurses, making temperature measurement standard

20. Monthly charts reporting on hypothermia distribution and data quality, doing Plan-Do-Study-Act circles continuously 
Table 2

Characteristics of VLBW infants in pre-QI and post-QI group

\begin{tabular}{|llll|}
\hline & $\begin{array}{l}\text { Pre-QI phase } \\
(\mathbf{n}=270)\end{array}$ & $\begin{array}{l}\text { Post-QI phase } \\
(\mathbf{n}=480)\end{array}$ & Pvalue \\
\hline $\mathrm{GA}\left[\right.$ weeks, $\left.\mathrm{M}\left(\mathrm{Q}_{1}, \mathrm{Q}_{3}\right)\right]$ & $29.6(28.3,31.4)$ & $29.7(28.3,31.0)$ & 0.954 \\
\hline $\mathrm{GA}<28$ weeks & $47(17.4)$ & $98(20.4)$ & 0.316 \\
\hline BW $\left[\mathrm{g}, \mathrm{M}\left(\mathrm{Q}_{1}, \mathrm{Q}_{3}\right)\right]$ & $1210(1000,1360)$ & $1200(1000,1368)$ & 0.937 \\
\hline BW<1000g & $58(21.5)$ & $117(24.4)$ & 0.368 \\
\hline Sex (boy) & $150(55.6)$ & $233(48.5)$ & 0.065 \\
\hline SGA & $40(14.8)$ & $64(13.3)$ & 0.573 \\
\hline Caesarean section & $221(81.9)$ & $373(78.5)$ & 0.278 \\
\hline Multiple birth (twins or more) & $57(21.1)$ & $113(23.5)$ & 0.445 \\
\hline Apgar score at 5 min $<7$ & $40(14.8)$ & $56(11.7)$ & 0.219 \\
\hline Intubation at delivery room & $80(29.6)$ & $135(28.1)$ & 0.662 \\
\hline Maternal hypertension & $124(45.9)$ & $182(39.9)$ & 0.113 \\
\hline Antenatal use of full course of steroid & $163(64.2)$ & $273(67.2)$ & 0.418 \\
\hline
\end{tabular}

Table 3

Admission temperature distribution among VLBW infants in pre-QI, and post- QI phases

\begin{tabular}{|llll|}
\hline & $\begin{array}{l}\text { Pre-QI phase } \\
(\mathbf{n = 2 7 0 )}\end{array}$ & $\begin{array}{l}\text { Post-QI phase } \\
(\mathbf{n = 4 8 0})\end{array}$ & P value \\
\hline Average admission temperature $\left[{ }^{\circ} \mathrm{C}, \mathrm{M}\left(\mathrm{Q}_{1}, \mathrm{Q}_{3}\right)\right]$ & $35.5(35.2,36.0)$ & $36.0(35.8,36.5)$ & $<0.001$ \\
\hline Hypothermia & $259(95.9)$ & $342(71.3)$ & $<0.001$ \\
\hline Mod-severe hypothermia & $185(68.5)$ & $144(30.0)$ & $<0.001$ \\
\hline Normothermia & $11(4.1)$ & $136(28.3)$ & $<0.001$ \\
\hline Hyperthermia & $0(0.0)$ & $2(0.4)$ & 0.288 \\
\hline
\end{tabular}


Table 4

Comparison of adverse outcomes among VLBW neonatess in pre-QI and post- QI phase

\begin{tabular}{|llll|}
\hline Outcome & $\begin{array}{l}\text { Pre-QI phase } \\
(\mathbf{n = 2 7 0 )}\end{array}$ & $\begin{array}{l}\text { Post-QI phase } \\
(\mathbf{n = 4 8 0})\end{array}$ & P value \\
\hline Composite outcome & $101(37.4)$ & $159(33.1)$ & 0.237 \\
\hline Mortality & $29(10.7)$ & $13(2.7)$ & $<0.001$ \\
\hline LOS & $89(33.0)$ & $105(21.9)$ & 0.001 \\
\hline NEC (Bell stage $\geq 2)$ & $14(5.2)$ & $13(2.7)$ & 0.081 \\
\hline IVH (Papile grade 3/4) & $6(2.2)$ & $17(3.5)$ & 0.314 \\
\hline pneumorrhagia & $17(6.3)$ & $18(3.8)$ & 0.113 \\
\hline BPD (moderate and severe) & $27(10.0)$ & $53(11.0)$ & 0.657 \\
\hline ROP (grade 3/4) & $3(1.1)$ & $7(1.7)$ & 0.754 \\
\hline
\end{tabular}


Table 5

Unadjusted and adjusted relative risk of outcomes for VLBW infants during post-QI phase with reference to pre-QI phase

\begin{tabular}{|c|c|c|c|}
\hline \multirow[t]{2}{*}{ Adverse outcomes } & \multirow[t]{2}{*}{$\begin{array}{l}\text { Pre-Ql phase } \\
(n=270)\end{array}$} & \multicolumn{2}{|l|}{$\begin{array}{l}\text { Post-QI phase } \\
(n=480)\end{array}$} \\
\hline & & Unadjusted RR (95\% Cl) & aRR (95\% Cl) \\
\hline Hypothermia & 1.000 & $0.105(0.056,0.199)$ & $0.103(0.054,0.194)$ \\
\hline Mod-severe hypothermia & 1.000 & $0.197(0.143,0.272)$ & $0.185(0.132,0.258)$ \\
\hline Composite outcome & 1.000 & $0.829(0.607,1.131)$ & $0.789(0.560,1.110)$ \\
\hline Mortality & 1.000 & $0.231(0.118,0.453)$ & $0.188(0.091,0.388)$ \\
\hline LOS & 1.000 & $0.569(0.408,0.795)$ & $0.557(0.397,0.782)$ \\
\hline NEC (Bell stage $\geq 2$ ) & 1.000 & $0.509(0.236,1.100)$ & $0.509(0.235,1.102)$ \\
\hline IVH or PVL (Papile grade 3/4) & 1.000 & $1.616(0.629,4.148)$ & $1.618(0.624,4.195)$ \\
\hline Pneumorrhagia & 1.000 & $0.580(0.294,1.145)$ & $0.558(0.279,1.117)$ \\
\hline BPD (moderate and severe) & 1.000 & $1.117(0.685,1.822)$ & $1.070(0.645,1.773)$ \\
\hline ROP (grade 3/4) & 1.000 & $1.508(0.397,5.734)$ & $1.420(0.371,5.439)$ \\
\hline \multicolumn{4}{|c|}{$\begin{array}{l}\text { Abbreviations: RR Relative ratio, CI Confidence interval, BPD Bronchopulmonary dysplasia, IVH } \\
\text { Intraventricular haemorhage, NEC Necrotizing enterocolitis, LOS Late-onset neonatal sepsis, ROP } \\
\text { Retinopathy of prematurity, aRR Adjusted for BW, GA,SGA, Apgar score at } 5 \text { min < } 7 \text { by binary logistic } \\
\text { regression analysis. }\end{array}$} \\
\hline
\end{tabular}

\section{Figures}




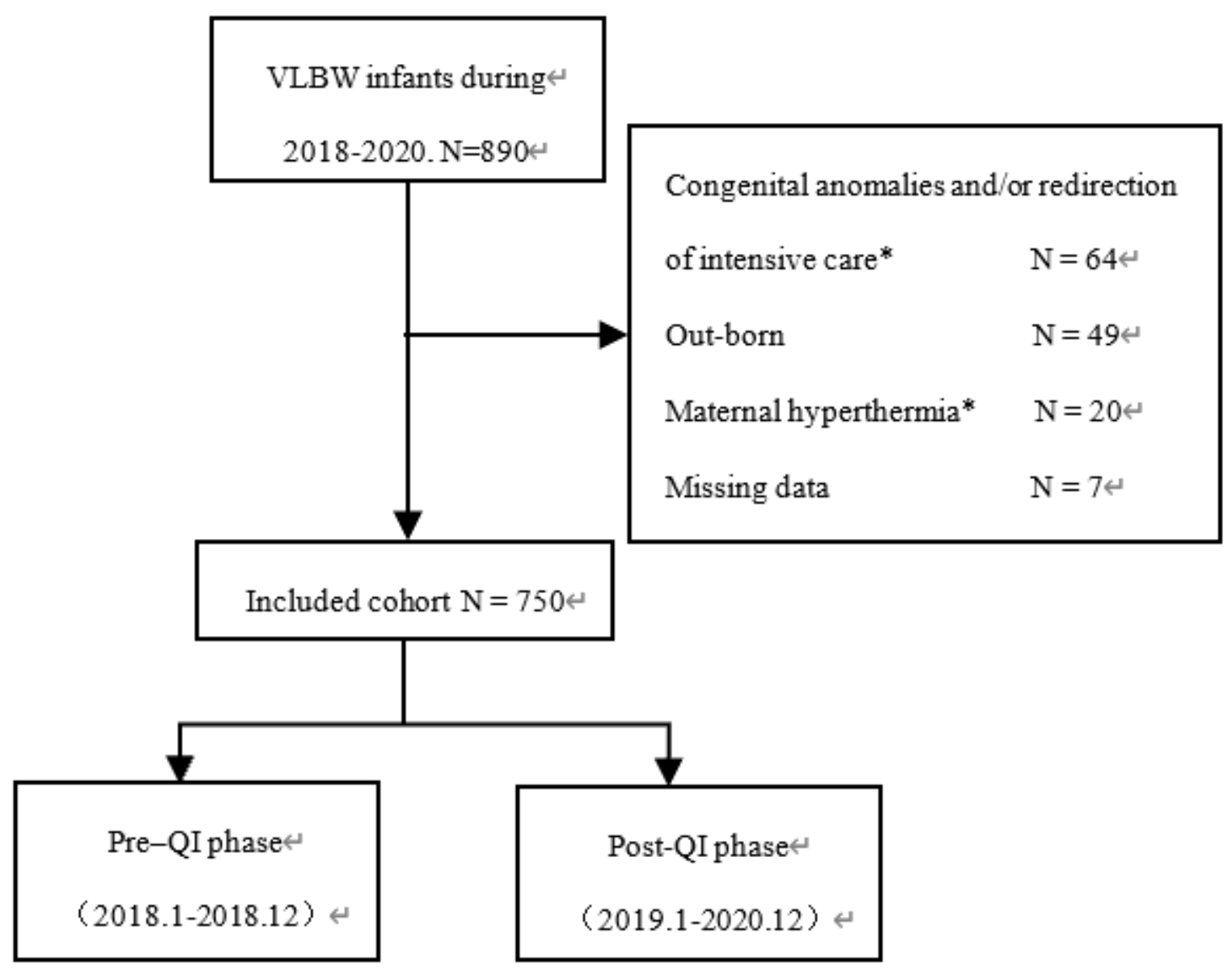

Figure 1

Patient Inclusion A total of 890 in-born infants with a BW $<1500 \mathrm{~g}$ were enrolled in the study; 49 infants were excluded because they were out-born; 20 infants were excluded because their mother had a fever during delivery (temperature $\geq 38.4^{\circ} \mathrm{C}$ ). Additionally, 64infants with redirection of intensive care and 7 infants with missing temperature data were excluded. The remaining 750 VLBWls were included in this analysis, 270 infants in pre-QI phase and 480 infants in post-QI phase, respectively. (*: limited care (not intensifying medical treatment) or withdrawal of care; Maternal hyperthermia*: mothers had a fever temperature $\left(\geq 38.4^{\circ} \mathrm{C}\right)$ during delivery). 


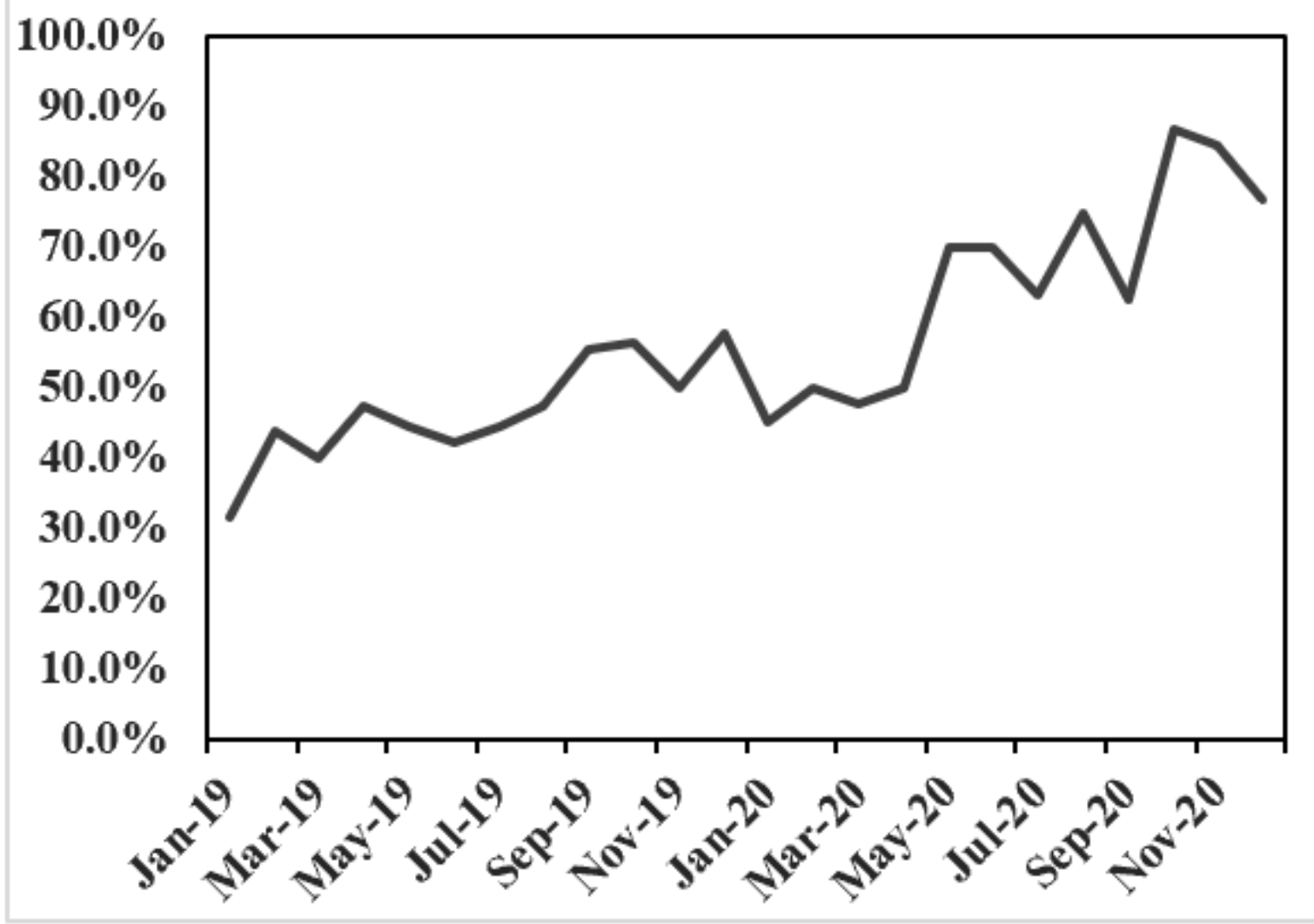

Figure 2

Month proportion of rectal temperature $\geq 36.5^{\circ} \mathrm{C}$ within first hour after admission during QI phase

\begin{tabular}{|l|l|l|l|l|}
\hline \multicolumn{5}{|c|}{ NICU temperature worklist for VLBW neonates } \\
\hline Patient name: & \multicolumn{1}{|l|}{ Birth date: } & Admission time: & Birth weight: & Gestational age: \\
\hline Temperature measurement & \multicolumn{3}{|l|}{} \\
\hline Recording time: & Temperature /C & Recording staff & Recorder signature & \multicolumn{1}{l|}{$\begin{array}{l}\text { If not record, explain } \\
\text { reason }\end{array}$} \\
\hline 10min after birth & & & & \\
\hline Arriving at NICU & & & & \\
\hline 30min after birth & & & & \\
\hline 60min after birth & & & & \\
\hline 90min after birth & & & & \\
\hline 120min affer birth & & & & \\
\hline 150min affer birth & & & & \\
\hline 180min afler birth & & & & \\
\hline
\end{tabular}

Figure 3 
The front side of worklist for VLBW neonates

\begin{tabular}{|c|c|c|c|c|}
\hline \multicolumn{5}{|c|}{ NICU temperature worklist for VLBW neonates } \\
\hline \multicolumn{5}{|c|}{ Interventions to keep warm, check yesino } \\
\hline 1 & Prenatal preparation (prenatal coesulation, forme maltidisciplinary team, chock materials) & yes $\square$ & no $\square$ & \\
\hline 2 & Set the ambient temperasure above $25^{\circ} \mathrm{C}$, set rabiant warmers a $34^{4} \mathrm{C}$ & yos $\square$ & m & $\sqsupset$ \\
\hline 3 & Prewarn the hat & yes $\square$ & no $\square$ & \\
\hline 4 & Repiec a polyethylene wrap & yes $\square$ & no $\square$ & \\
\hline 5 & 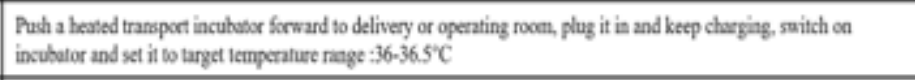 & yes $\square$ & no $\square$ & \\
\hline 6 & Prebentad blankets woęping after birth & yes $\square$ & no $\square$ & \\
\hline 7 & Quickly weight afler being plased in a pre-warmed blanket & yes $\square$ & no $\square$ & \\
\hline 8 & Lefant immediately after birth wapped with a polyellylene wrap without drying & yes $\square$ & no $\square$ & \\
\hline 9 & Place a pre-warmod hat on the head & yos $\square$ & Do $\square$ & \\
\hline 10 & Reswscitasion under chentical pretheared manress & yes $\square$ & no $\square$ & \\
\hline 11 & Document temperature at 10 min after birth & y $\square$ & no $\square$ & \\
\hline 12 & Pur infints into a hearod trassport incubator and start trassportation & yos $\square$ & no $\square$ & \\
\hline 13 & Preben daily matterials in isculuncer (diapers, axygen probe, stethoscope) & yes $\square$ & no $\square$ & \\
\hline 14 & 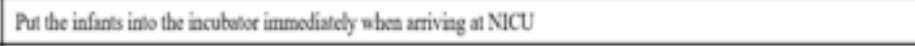 & yos $\square$ & no $\square$ & \\
\hline 15 & Document vemperanture continuosely within one hoser after birh & yes $\square$ & no $\square$ & \\
\hline 16 & Retest vemperature when arriving at the NICU, soon aflet every 30 mi & yes $\square$ & no $\square$ & \\
\hline 17 & Document the time point when temperanure a巴 $365^{\circ} \mathrm{C}$ & yes $\square$ & no $\square$ & \\
\hline 18 & Nuring and medical operations are centralized implemented & yes $\square$ & no $\square$ & \\
\hline 19 & Training and assesments oa temperature measurement for narses, making temperature measurement standard & yes $\square$ & m & \\
\hline 20 & Montly charts reporting on hypobhermia distribution and danta quality, doing Plan-Do-Shady-Act circles continuously & yes $\square$ & no $\square$ & \\
\hline
\end{tabular}

Figure 4

The back side of worklist for VLBW neonates 


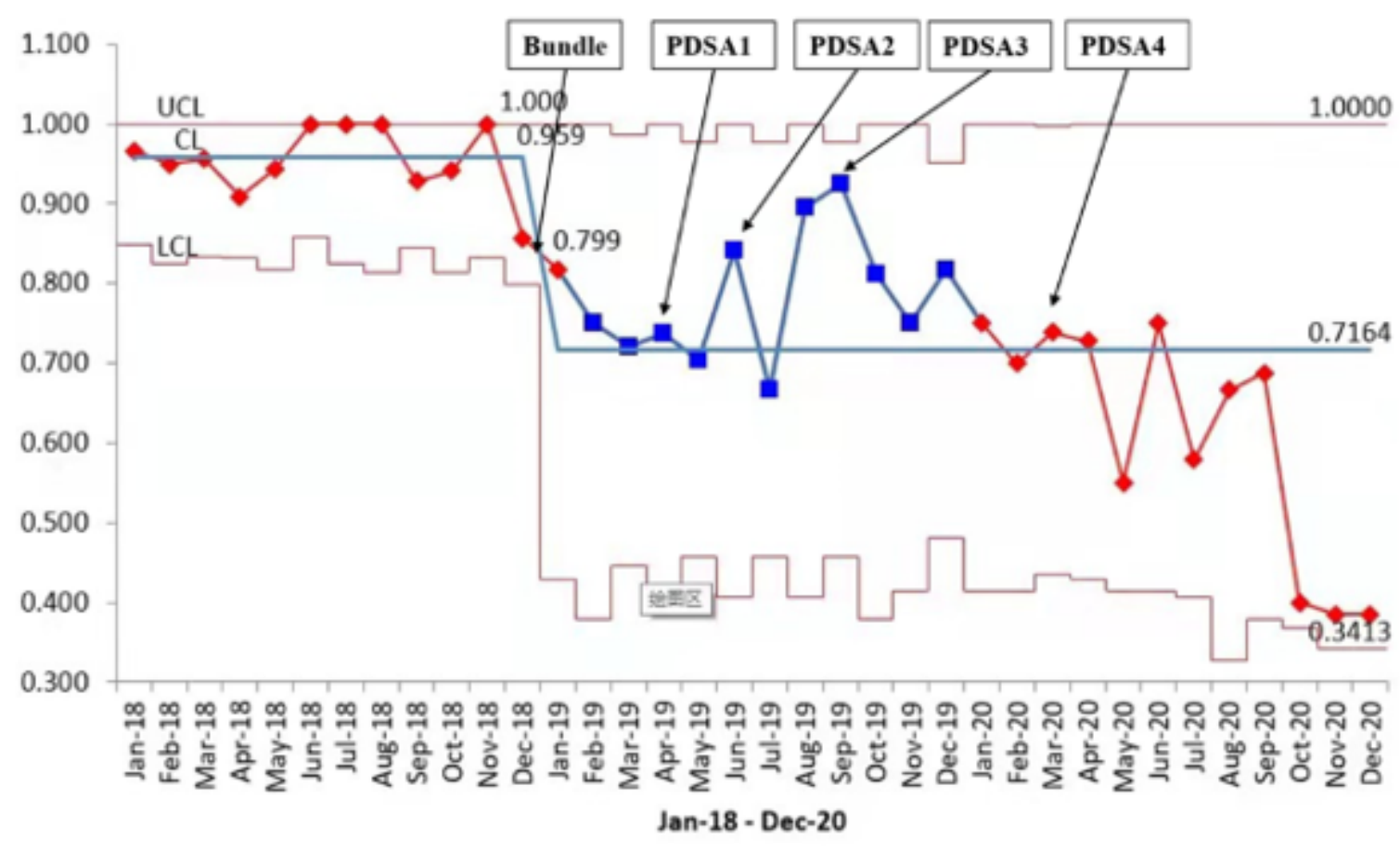

\section{Figure 5}

P-chart of monthly AH percentage in January 2018-December 2020, Subdivided into pre-QI period and post-QI period. CL, center line; LCL, lower control limit; UCL, upper control limit. Arrows show change of major interventions including the thermoregulation bundle: Initial Bundles (January 1-March 31, 2019); PDSA Cycle 1 (April 1-May 31, 2019): Using polyethylene occlusive wrap infants without drying instead of drying infants immediately after birth. PDSA Cycle 2 (June 1-August 31, 2019): a heated transport incubator introduction. PDSA Cycle 3 (September 1,2019 - March 31, 2020): Revise admission hypothermia check list to supervise effectively. PDSA Cycle 4 (April 1- December 31, 2020): Various online education lectures monthly to build up confidence, faith and further emphasize heat preservation awareness. 